\title{
QUALITY CHECK OF CRISIS MAPS PRODUCED OVER FIVE YEARS BY COPERNICUS EMS
}

\author{
K. Spasenovic ${ }^{1}$, D. Carrion ${ }^{1 *}$ \\ ${ }^{1}$ DICA - Geodesy and Geomatics, Politecnico di Milano, Milan, Italy - (katarina.spasenovic, daniela.carrion) @ polimi.it
}

Commission III, ICWG III/IVb

KEY WORDS: crisis map, quality assessment, readability, metadata

\begin{abstract}
:
Crisis mapping is a widely used tool for the digital representation of a disaster situation. It provides stakeholders with spatial information of the crisis describing type and severity of damages, impact on the area and its population. The quality of crisis maps is of a great importance, since the disaster response depends on the provided information. In some cases, in order to deliver a map on time, its quality can be lower than expected. The evaluation of a big sample of rapid maps produced between 2013 and 2017 and collected from open source Copernicus service have been performed. The quality check has been performed by visual analysis, observing around 36 parameters defined in the validation protocol designed at the Joint Research Centre (JRC) of the European Commission. Overall conclusion is that crisis maps produced during the observed period have shown good level of the quality, that can satisfy the need of user. Still, there are some parameters that could be delivered with more details and precision. The results are analysed in details and remarks are presented.
\end{abstract}

\section{INTRODUCTION}

Rapid Mapping has a great role during a crisis event, because it provides in a short time (within the first 24-72 hours) geospatial and thematic information related to the situation, which will be used for supporting emergency management activities. During the last decades, hundreds of maps have been produced every year representing disasters happening worldwide. The quality of crisis maps is of a great importance, since the disaster response depends on the provided information. The process of map production is generally based on exploitation of satellite images (Voigt et al. 2007). Main topographic features representing the pre-event situation should be provided (e.g. transportation network, build-up, land cover, hydrography, and utilities) in order to form better general image of the territory to understand the event impact and plan the interventions. As stated before, time is crucial for Emergency Management (EM), to minimize the time required for post-event map production, Rapid Mapping should be based on consolidated operational workflows. Ajmar et al. (2015) have proposed a simplified workflow highlighting the main processing steps: (1) Pre-event preparation activities (Reference data regarding the affected area before the event), (2) Event (Up-to-date reference data covering the affected area before the event), (3) Reference data updating (Satellite/aerial post-event acquisition), (4) Satellite/aerial post event image processing, (5) Map production. There are more than one service providers involved in disaster Rapid Mapping such as United Nations UNOSAT rapid Mapping service, International charter space and major disasters, the European Union's Earth Observation Programme Copernicus Emergency Management service (EMS). Even though all previously presented Rapid Mapping providers are independent services, they collaborate in a partnership with each other and with other agencies and organisations of interest, in order to deliver good quality products in time.

The quality evaluation of the crisis maps ensures that all elements of the product meet the required level of accuracy and completeness. The needs of end-users should be satisfied, information provided with crisis maps should have certain level of details and should be understandable as well as suitable for additional manipulations.

In this paper, a sample of crisis maps produced between 2013 and 2017 has been evaluated through a list of parameters selected from the validation protocol designed at the Joint Research Centre (JRC) of the European Commission (EC) (Broglia et al. 2010, Corbane et al. 2011a). The analysis focuses on Copernicus Emergency Management Service (EMS), which is implemented by the EC and which provides crisis and reference maps and preand post-disaster situation maps to entities and organisations working for emergency management. In this research, a full validation of the maps has not been performed, because it would have required the availability of ground truth or, more in general, reference data, for a very large number of events. The target of the evaluation was to explore the crisis maps data quality, overviewing a large number of crisis maps over five years period, applying cost and time effective approach instead of in-depth approach.

\section{COPERNICUS EMERGENCY MANAGEMENT SERVICE}

Copernicus EMS shares its products through an online platform, which is open to all users, providing geospatial information for emergency response related to different types of disasters worldwide. The service is based on the acquisition, processing and analysis, often in rapid mode, of satellite imagery and other geospatial raster and vector data sources. Copernicus EMS produces two types of maps: Rapid Maps and Risk Recovery Maps. For the purpose of the research presented in this paper, the focus will be on Rapid Maps. The service has been operationally active since April 1st, 2012. Search tool of the service allows selection of the maps produced only over the last five years, maps dating from earlier years could be found searching database manually. It is very important to emphasize that even though the

\footnotetext{
${ }^{*}$ Corresponding author
} 
maps are standardized and Copernicus Rapid Mapping team covers all the processing steps presented in the workflow by Ajmar et al. (2015), they perform as well the product quality check before the distribution (see Copernicus EMS Mapping Manual of Operational Procedures, EC 2015). The Copernicus EMS Mapping Validation is carried out independently from map production and it is based on the validation protocol developed at the Joint Research Centre (JRC), presented by Broglia M., et al. (2010).

\section{THE SAMPLE OF CRISIS MAPS}

When data have been acquired to perform the analysis presented in this paper, the oldest crisis map available with Copernicus search tool dated from 2013. The first event considered is the Flooding of refugee camp in Mafraq, Jordan that occurred on $7^{\text {th }}$ of January 2013. The last considered event is Flood in Lower Saxony, Germany that happened on $14^{\text {th }}$ of December 2017. The types of events covered by Copernicus service during the period of interest were fifteen. Among those, nine types of events, considered as the most frequent ones, have been considered for the selection of a map sample for this work. For those selected types of events, over the period of interest (2013-2017), Copernicus EMS has been activated 285 times and produced 2635 crisis maps in the Rapid Mapping mode. For this analysis, $30 \%$ of total downloaded maps, corresponding to a set of 760 maps, has been extracted and considered as a representative sample on which further assessment has been performed. In order to obtain a representative sample, among all selected activations, one map has been selected per every affected zone of each activation. The congruence of the sample of maps with respect to the 2013-2017 population was confirmed by comparing the maps distribution per event and per continent over the observed period. In Figure 1, the sample distribution over time and per type of event is presented. It can be noticed that the total number of maps per year grows over time: the number of produced maps in 2017 was five times more than in 2013. It could be seen in the pie chart that the most dominant type of event was flood (42\% of representative sample), followed by fire and earthquake (20\% and $14 \%$ of sample, respectively).

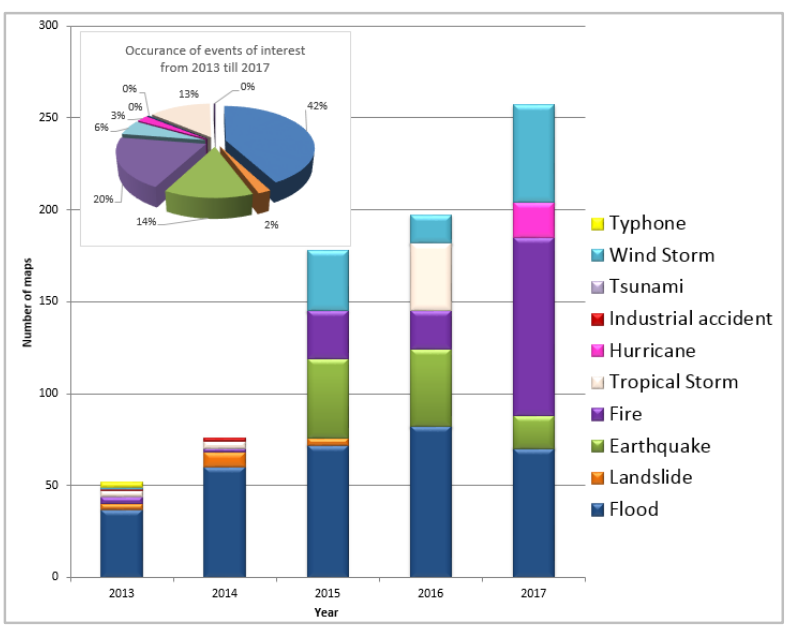

Figure 1. Distribution of the maps considered as representative sample over the time per type of the event

Rapid maps could be delivered in three standardized forms: reference, delineation and grading map. The representative sample had $21 \%$ of referenced maps, $45 \%$ of delineation and $34 \%$ of grading maps. It was good that majority of sampled maps were delineation and grading, so the quality of crisis representation in digital format could be better checked.

\section{QUALITY ASSESSMENT OF CRISIS MAPS}

Information presented in the map should be clear, complete and provided together with metadata ${ }^{1}$. Complete information provided by crisis maps would allow all users to contribute the emergency actions in the best way, improving the response quality. When fundamental details are missing from geographic data, such as scale and information sources' resolution, or when it is incomplete, for example when the legend is not clear enough, often it is not possible to exploit them, e.g. for integration with other layers into Geographic Information Systems (GIS) (Carrion et al. 2013). In general the quality of data is defined as ,fitness for use ee and some parameters commonly used to measure it are positional and thematic accuracy, consistency and completeness (US National Committee Digital Cartographic Data Standards Task Force (DCDSTF), 1988). According to Borrough and McDonnell (2000) the factors that affect spatial data quality are:

- Currency (nowadays maps are in electronic format that makes them out-dated; data provided can be used in a long period of time)

- Completeness

- Consistency (connection and dependence between data)

- Accessibility (easy access to the data information)

- Accuracy and precision (correctness of provided data)

- Source of errors in original data

- Source of errors in derived data and in the results of modelling and analysis

These quality parameters have been taken into account in the validation protocol (Broglia et al. 2010, Corbane et al. 2011b) for crisis maps, which has been developed at the JRC and applied, at first, in the framework of SAFER FP7 project (Denis et al. 2009, Carrion et al. 2011). Quantitative and qualitative parameters of the protocol have been grouped into four categories: reliability of the information content, consistency of the information support, usability of the product and efficiency of the service.

\subsection{The quality assessment checklist}

The aim of the work presented in this paper was to explore the crisis maps data quality with a fast and global approach. A full validation of a large sample of maps, including the evaluation of spatial and thematic accuracy, would bring challenges of finding needed information and data connected to the events all around the world. Consequently, the evaluation process relied on the idea to analyse a subset of quality parameters, considering core requirements needed for further utilization of crisis maps. Some examples of these core requirements are the possibility to understand the geographic position and time of the disaster, the presence of a clear legend, the presence of complete map metadata, including key elements such as declaration of accuracy and scale. Guided by the parameters presented in the JRC validation protocol, a more synthetized version of evaluation framework has been designed, based also on a previous quality check of crisis maps (Carrion et al., 2013). Parameters that can be verified by a visual analysis have been considered and finally adapted to the purpose. The biggest attention has been devoted to the maps readability and usability. Table 1 shows the final quality

\footnotetext{
${ }^{1}$ Metadata is a set of data that that describes and give information about the data.
} 
assessment checklist presenting the 36 parameters that have been controlled during the quality assessment process.

\begin{tabular}{|c|c|}
\hline Category & Parameter \\
\hline \multirow{9}{*}{$\begin{array}{l}\text { Usability } \\
\text { general } \\
\text { information }\end{array}$} & Service provider \\
\hline & Type of crisis event \\
\hline & Type of map \\
\hline & Date of crisis (month and year) \\
\hline & Place of crisis event - continent \\
\hline & Place of crisis event - country \\
\hline & Place of crisis event - town \\
\hline & First language of the map \\
\hline & Second language of the map \\
\hline \multirow{3}{*}{$\begin{array}{l}\text { Reliability of } \\
\text { the information } \\
\text { content }\end{array}$} & $\begin{array}{l}\text { Information on occlusion of EO sources } \\
\text { (clouds) }\end{array}$ \\
\hline & $\begin{array}{l}\text { Time gap between crisis event and crisis } \\
\text { map production }\end{array}$ \\
\hline & $\begin{array}{l}\text { Legend semantic definition for thematic } \\
\text { data (e.g. Corine Landcover) }\end{array}$ \\
\hline \multirow{2}{*}{$\begin{array}{l}\text { Consistency of } \\
\text { the additional } \\
\text { information }\end{array}$} & $\begin{array}{l}\text { Consistency between map and legend } \\
\text { symbols }\end{array}$ \\
\hline & $\begin{array}{l}\text { Consistency between declared scale and } \\
\text { resolution of the images used to produce } \\
\text { the map }\end{array}$ \\
\hline \multirow{12}{*}{$\begin{array}{l}\text { Usability of } \\
\text { product }\end{array}$} & $\begin{array}{l}\text { Contrast between background and } \\
\text { thematic entities }\end{array}$ \\
\hline & Symbols easily differentiable \\
\hline & Scale bar \\
\hline & Declared nominal scale \\
\hline & Presence of overview map \\
\hline & Coordinate graticules/grid and its labels \\
\hline & Presence of interpretation text \\
\hline & Presence of map title \\
\hline & $\begin{array}{l}\text { Completeness of title: information on } \\
\text { geographical area, date of event, thematic } \\
\text { content }\end{array}$ \\
\hline & Type of map background \\
\hline & $\begin{array}{l}\text { Information on conditions related to } \\
\text { access, use and information sharing }\end{array}$ \\
\hline & $\begin{array}{l}\text { Responsibility assumption (on data set or } \\
\text { information sources) }\end{array}$ \\
\hline \multirow{10}{*}{$\begin{array}{l}\text { Usability } \\
\text { metadata }\end{array}$} & $\begin{array}{l}\text { Metadata - Description of data sources } \\
\text { used }\end{array}$ \\
\hline & Metadata - description of processing steps \\
\hline & $\begin{array}{l}\text { Metadata - information on quality control } \\
\text { procedure used }\end{array}$ \\
\hline & $\begin{array}{l}\text { Metadata - information on known sources } \\
\text { of error }\end{array}$ \\
\hline & Metadata - information on spatial accuracy \\
\hline & $\begin{array}{l}\text { Metadata - information on thematic } \\
\text { accuracy }\end{array}$ \\
\hline & Metadata - point of contact \\
\hline & Metadata - reference datum \\
\hline & Metadata - reference projection \\
\hline & Metadata - coordinate system \\
\hline
\end{tabular}

Table 1. List of 36 parameters considered for the rapid map quality check

\section{DISCUSSION AND RESULTS}

An effective quality assessment must be consistent and as much objective as possible. After selecting the quality assessment checklist, it was necessary to ensure uniformity in terminology in order to minimize the mistakes in data entry. For that reason a range of possible answers for every parameter have been defined (e.g., Yes, Partial, No). Each parameter from the assessment checklist was evaluated on the representative sample of 760 crisis maps and the most suitable answer have been selected from the drop-down menu. The maps have been observed in digital format. During the evaluation process, it was considered that maps should be understandable for all users (Carrion et al. 2011), including both expert and non-expert people. The obtained results are grouped per category and presented in the following paragraphs.

\subsection{Usability of general information}

When a user starts to use the map it is very important that he is able to clearly understand the main information related to the event and map production. An effective and very short summary of the event is present in the title and, possibly, in the subtitle. After evaluating all parameters that are providing the general information about the event, it has been found out that only 4 maps out of 760 were missing the date of crisis event, while all other parameters from the group were always present.

\subsection{Reliability of the information content}

Reliability refers to how much the user is confident with the information that he is getting from the product. In the following text, the crisis map's parameters that could be used for the evaluation of a product reliability by visual interpretation will be presented.

Information on occlusion of EO sources (clouds) is essential for understanding legibility and reliability of the content. Crisis maps are produced on the basis of satellite images (Voigt et al. 2007), where clouds could be an obstacle for the interpretation of the crisis on the territory. Information about clouds could be shown as a percentage of the spatial extent of the mapped area and/or it could be directly represented on the map (i.e. occluded areas represented with a mask and defined in the legend, Broglia M. et al. 2010). The assessment of the presence of information about cloud coverage gave the result that $46 \%$ of observed maps have significant (more than 30\%) presence of clouds, showing that almost half of the sample have had this type of obstacle that could be a limitation in the interpretation of the situation on the ground.

When speaking of time gap between the event and map production, the smaller is the gap, the more information is reliable. The time of map production depends the most on the satellite data acquisition, it is not always the case that satellite images covering the area of interest are available right after the event. The sample has shown that $8 \%$ of maps have been produced at the day of the event, 29\% (highest peak) within first 72 hours. It must be considered that not all the crisis maps are requested in rapid mode and they can be updated by the service provider at a later stage. With respect to the sampled maps, during the first two weeks, high production of crisis maps has been recorded, after that the production decreased, see Figure 2. 


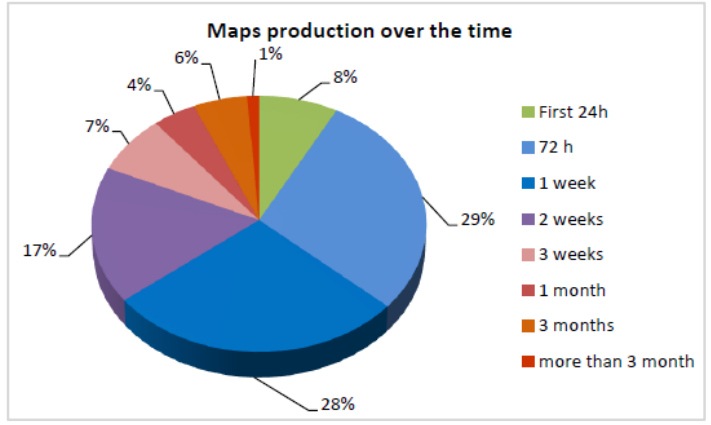

Figure 2. Pie chart of the time gap between crisis event and map production

\subsection{Consistency of the additional information}

A product is, in general, considered consistent when it does not contain contradictions that could be observed by comparing different elements of the same product. For example, the map and the legend should contain a consistent set of symbols (Golebiovska 2011). Symbols used in the map should be present in the legend and vice versa. After performing the assessment, it was found that $89 \%$ of maps were satisfying consistency parameters (see Table 2).

\begin{tabular}{|c|c|c|c|c|c|}
\hline & Present & $\begin{array}{c}\text { Partial } \\
\text { (missing } \\
\text { some } \\
\text { symbols } \\
\text { in the } \\
\text { map) }\end{array}$ & $\begin{array}{c}\text { Partial } \\
\text { (missing } \\
\text { some } \\
\text { symbols } \\
\text { in the } \\
\text { legend) }\end{array}$ & $\begin{array}{c}\text { No } \\
\text { (missing } \\
\text { symbols } \\
\text { in the } \\
\text { map) }\end{array}$ & $\begin{array}{c}\text { No } \\
\text { (missing } \\
\text { symbols } \\
\text { in the } \\
\text { legend) }\end{array}$ \\
\hline $\begin{array}{c}\text { No. of } \\
\text { maps }\end{array}$ & 675 & 39 & 1 & 39 & 6 \\
\hline$\%$ & 89 & 5 & 0 & 5 & 1 \\
\hline
\end{tabular}

Table 2 Presence of consistency between map and legend symbols

The consistency of a crisis maps could be checked also by comparing the declared scale and the resolution of the images used for the map production. The representation scale could be declared with scale bar or by numeric ratio (e.g. 1:100,000). Adequacy of information sources with the declared nominal scale could be verified with the resolution of the imagery that can be found in the general information of the map layer e.g. GSD ${ }^{2}$ $0.5 \mathrm{~m}$. Having information about the resolution, it was possible to calculate the range of map scale in which the map could be represented (American Society for Photogrammetry and Remote Sensing (ASPRS) Specifications Standards Committee 1990). Analysing this parameter, $70 \%$ of products were consistent with respect to the declared scale and resolution.

\subsection{Usability of the product}

Usability of product is a crucial aspect of the validation and quality assessment, since its aim was narrowing the gap between the service provider and the end user. The usability of crisis maps includes reading, interpreting, analysing and integrating information about the event provided by a map. For example, differentiability of symbols is related to the size, orientation, arrangement and colour. If symbols are overlapping it is very difficult to understand what they are presenting. The results have shown the presence of symbols that were overlapping in $9 \%$ of the sampled maps. The majority of maps (80\%) had easily differentiable symbols and $1 \%$ of total has hard to distinguish symbols. All the other parameters contributing to the usability of the product have shown to be present in almost all maps. In addition, 750 maps out of 760 had complete map title, while the remaining 10 crisis maps were missing one of the title elements.

Often, the background of crisis maps is represented by satellite images. A topographic map as a background, could be in most cases preferable (Carrion et al., 2013), because it corresponds to interpreted content. According to the work presented by Konecny et al. (2011) the efficiency of the data interpretation does depend on the background map.

Observing the map background, it was found that $21 \%$ of the checked maps had a topographic map as a background, while most of maps $(76 \%)$ had a satellite image as a background. The presence of DEM (Digital elevation model) as a background was observed for flood events only, in particular, they were used in $2 \%$ of the flood maps (Figure 3). In Figure 3, the type of background with respect to the type of event is shown. It is possible to observe that landslide and fire events were almost equally presented with satellite and topographic maps. For all other events satellite image was present in more than $70 \%$ of the maps.

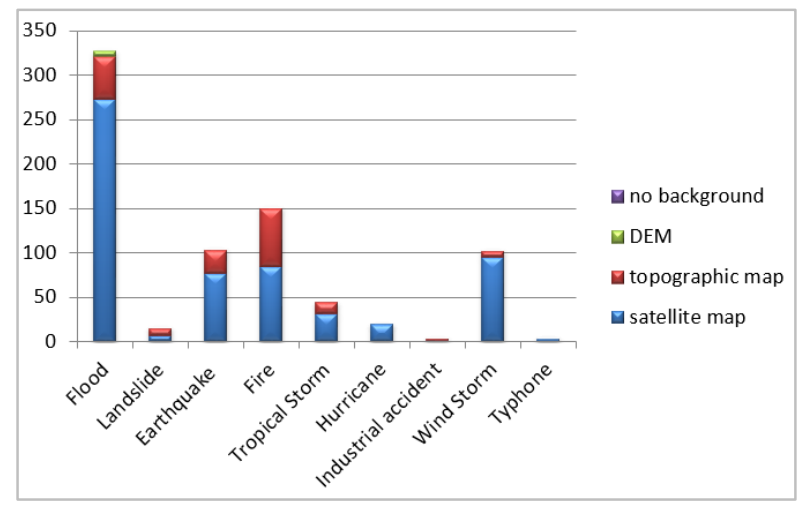

Figure 3. The use of different map's background with respect to the type of event

\subsection{Usability of metadata}

Metadata in general is additional information of the product data and it is necessary and fundamental to help the user understand if the product suits his purpose. Parameters connected to metadata (presented in the quality check list in Table 1) have been evaluated and it has been find out that $4 \%$ of maps missed completely description of data source used, $6 \%$ missed the description of processing steps, and $12 \%$ of sampled maps did not have information about known source of error (thematic accuracy). Spatial accuracy was the weakest parameter, since $48 \%$ of maps did not have any information about spatial accuracy and $15 \%$ had it but it was not complete. The rest of parameters related to metadata satisfied all criteria and were presented well in almost all sampled maps.

\subsection{Evaluation of the parameters completeness over time}

It can be interesting to observe the performance of the assessment parameters over time on the sampled maps. The evaluation over

\footnotetext{
2 GSD stands for Ground sample distance. It is used in a digital photo of the ground from air or space and it represents the distance between the centers of adjacent pixels measured on the ground (Kapnias D. et al 2008).
} 
time could help to see the evolution, if any, of the parameters over the years and to understand if significant changes happened for a specific year. Parameters which showed a significant change over time were singled out and presented in Figure 4.

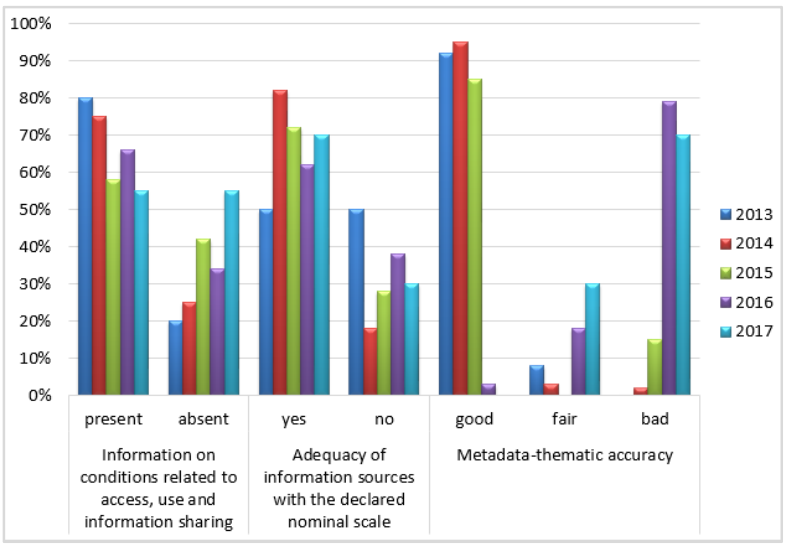

Figure 4. Evolution of quality parameters over time

The parameter presenting information related to access, use and sharing has recorded a similar behaviour over time, with a slight worsening. The highest numbers of maps having the information regarding the access were recorded during the first two years of the observed period and in later years, a decreasing has been recorded. Looking at the results representing the Consistency of the information support, the parameter about adequacy of information sources with the declared nominal scale shows that $70 \%$ maps were suitable, taking closer look on suitability dispersion per year it can be noted that it is very randomly distributed, and it changed from year to year. Thematic accuracy parameter has shown an increment in the number of maps with bad information: in the last two years it has been recorded that many maps were missing this information or very general statement was provided.

\subsection{Comparison of two studies}

Results obtained in this work have been compared with the results presented in a previous study, by Carrion et al. (2013), where a sample of maps produced between 2005 and 2010 by the world's leader providers had been evaluated through around 40 parameters. The 2012 study was performed before Copernicus EMS became operational. Copernicus service does perform quality check of crisis maps before distribution. Progress can be noticed by comparing the quality assessment regarding the period from 2005 to 2010 (Figure 5) with the quality assessment for the period from 2013 until 2017 (Figure 6). Large advances in the delivery of metadata information have been recorded, all parameters that were not satisfactory in the previous assessment have been improved although space for progress is present. Comparing Figures 5 and 6 it is evident that the presence of metadata regarding thematic accuracy has increased significantly, although almost half of the sampled maps of the most recent quality check are still missing this information. Exploring more in detail this parameter, it has been discovered that until the end of 2015, information regarding thematic accuracy was present. The accuracy value was declared, and the method of its assessment was stated. In the later delivery form, after the end of 2015, thematic accuracy information was missing or there was very general statement about the limitations related to it.

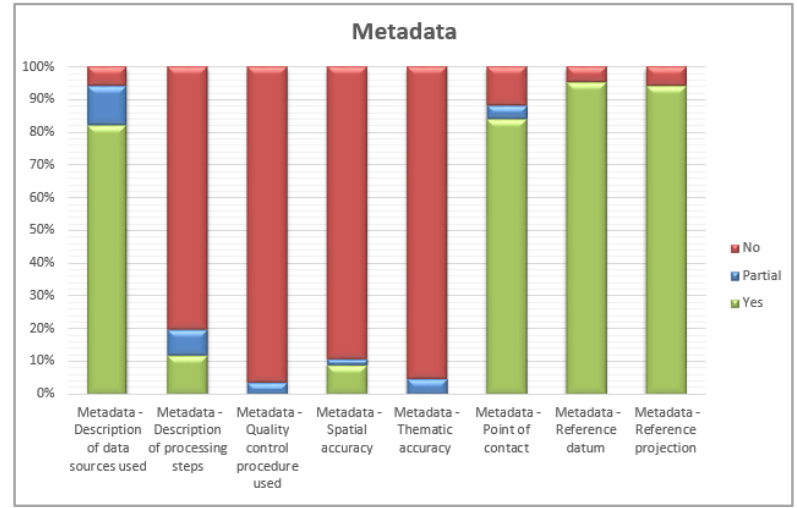

Figure 5. Presence of information regarding metadata from 2005 to 2010 (Carrion et al. 2013)

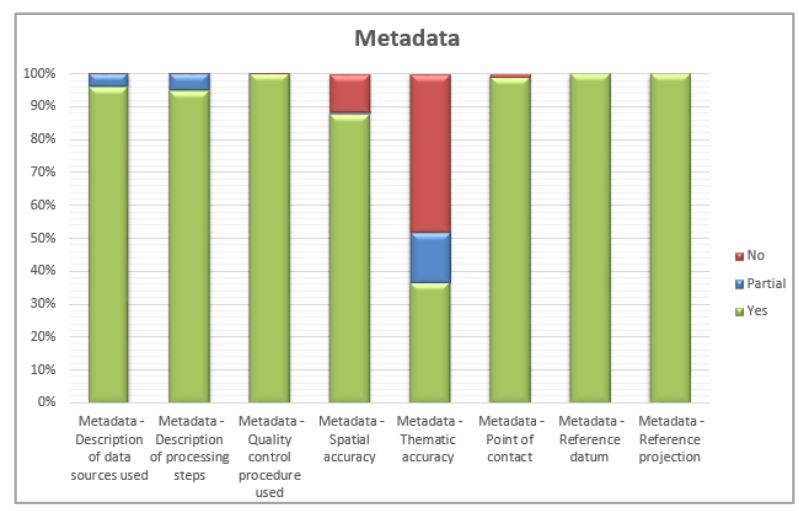

Figure 6. Presence of information regarding metadata from 2013 to 2017

\section{CONCLUSION}

A quality check of crisis maps produced from 2013 to 2017 has been performed examining a representative sample of 760 maps produced by Copernicus EMS. The assessment is based on visual analysis and on a set of parameters derived from the JRC validation protocol for emergency geo-information products. The idea of this paper is to provide with an overview of basic quality parameters over a large sample of crisis maps, without the availability of ground truth or reference data, thus not performing a full validation.

It is evident that, in most of cases, the lack in the product quality that have been singled out are related to the time constrains which crisis map service providers have to face. Even though, there are cases where more attention could be paid to the map itself, as a cartographic product. For example, inconsistencies between symbols presented in the map and legend, or between image resolution and map scale should not be present. Metadata are crucial to help to the user to understand if the map suits to the desired purpose, having in mind a variety of user's expertise. Nevertheless, some parameters could be delivered with more details and precision. Crisis maps produced during the observed period have shown a good level of quality, in general. It has been mentioned that Copernicus service does perform quality check of crisis maps before distribution, a higher level of quality can be noticed by comparing the quality assessment of the sample produced from 2005 to 2010 with respect to the previous quality assessment of maps produced from 2013 to 2017. In particular, large improvements in metadata information delivery have been recorded. In addition, most of the sampled maps had well 
explained processing steps. Over every delivered map there is information about the release inspector which has performed the quality control. This information could be very useful and helpful for increasing the map reliability. The majority of parameters that were not satisfactory in the assessment performed in 2013 have improved. A good example are metadata about spatial and thematic accuracies that have made an evident progress, although space for improvements is still present

Being a tool for representing and communicating the disaster situation to the stakeholders, crisis maps have a great role during the emergency management. It is very important to monitor the quality of such product and to keep the track of the attention that service provider is payed to map readability and metadata provision.

\section{REFERENCES}

Ajmar A., Boccardo P., Disabato F., Tonolo F.G., 2015. Rapid Mapping: geomatics role and research opportunities, Geodesy and geomatics to the edge: Springer. doi.org/10.1007/s12210015-0410-9.

American Society for Photogrammetry and Remote Sensing (ASPRS) Specifications Standards Committee, 1990. ASPRS accuracy standards for large-scale maps.Photogrammetric Engineering and Remote Sensing, 56 (7), 1068-1070.

Borrough P.A., McDonnell R.A., 2000. Principles of geographical information systems, Oxford University Press, Oxford.

Broglia M., et al., 2010. Validation protocol for emergency response geo-information products, JRC Scientific and Technical Reports, Luxembourg, Office for Official Publications of the European Communities. EUR Number: 24496 EN, ISBN 978-92-79-16428-6, doi.org/10.2788/63690.

Carrion D., Corbane C., Broglia M. and Pesaresi M., 2013. First extensive and cost-effective quality check of Crisis Maps: presentation of assessment parameters and results, International Journal of Digital Earth, 50-66. doi.org/10.1080/17538947.2012.674563.

Carrion D., Corbane C., Judex M., Broglia M., 2011. Crisis maps validation and user requirements: an experience in SAFER project, Gi4DM, Antalya Turkey: ISPRS.

Corbane C., Carrion D., Broglia M., 2011a. Development and implementation of a validation protocol for crisis maps: reliability and consistency assessment of burnt area maps. International Journal of Digital Earth, 8-24.

Corbane C., Pesaresi M., Carrion D., 2011b. Validation of EO-derived information for crisis management: A Digital Earth perspective in the VALgEO expert community, International Journal of Digital Earth, 1-7.

DCDSTF (Digital Cartographic Data Standards), 1988. The proposed standard for digital cartographic data, The American Cartographer, 9-140.

Denis G., Hello D., 2009. SAFER - Services and Applications for the Emergency Response: en route for a safer world with the GMES operational services, EGU General Assembly 2009, Vienna, Austria: 13454.
DG GROW, DG ECHO, DG JRC (European Commission), 2015. Copernicus Emergency Management Service Mapping Manual of Operational Procedures.

Golebiowska I., 2011. Strategies of legend and map integration, 25th International Cartographic Conference, Paris, 3-8.

Kapnias D., Milenov P., Kay S., 2008. Guidelines for Best Practice and Quality Checking of Ortho Imagery, JRC Scientific and Technical Reports, Luxembourg, Office for Official Publications of the European Communities. doi.org/10.2788/36028.

Konecny M. et al., 2011. The usability of selected base maps for crises management - users 'perspectives, Applied Geomatics, 189-198. doi.org/10.1007/s12518-011-0053-1.

Voigt S., Kemper T., Riedlinger T., Kiefl R., Scholte K., Mehl H., 2007. Satellite Image Analysis for Disaster and CrisisManagement Support, IEEE Transactions on Geoscience and Remote Sensing, vol. 45, no. 6, 1520-1528. doi.org/10.1109/TGRS.2007.895830. 